\title{
Depolarization-initiated endogenous cannabinoid release and underlying retrograde neurotransmission in interneurons of amygdala
}

\author{
Sodikdjon A. Kodirov, ${ }^{1,2}$ Kathrin Bonni, ${ }^{1}$ Michael Wehrmeister, ${ }^{1}$ and Beat Lutz ${ }^{1}$ \\ ${ }^{1}$ Institute of Physiological Chemistry and Pathobiochemistry, University Medical Center Mainz, 55099 Mainz, Germany; ${ }^{2}$ Center for \\ Biomedical Studies, Department of Biological Sciences, University of Texas at Brownsville, Texas 78520, USA
}

\begin{abstract}
The depolarization is also important for the short-term synaptic plasticity, known as depolarization-induced suppression of excitation (DSE). The two major types of neurons and their synapses in the lateral nucleus of amygdala (LA) are prone to plasticity. However, DSE in interneurons has not been reported in amygdala in general and in LA in particular. Therefore, we conducted the patch-clamp experiments with LA interneurons. These neurons were identified by lack of adaptation in firing rate of action potentials. In this study, we show for the first time a transient suppression of neurotransmission at synapses both within the local network and between cortical inputs and interneurons of the LA. The retrograde neurotransmission from GABAergic interneurons were comparable with that of glutamatergic pyramidal cells. That is the axonal terminals of cortical inputs do not posses selectivity toward two neuronal subtypes. However, the DSE of both types of neurons involve an increase in intracellular $\mathrm{Ca}^{2+}$ and the release of endogenous cannabinoids (eCB) and activation of presynaptic CBI receptors. The magnitude of DSE was significantly higher in interneurons compared with pyramidal cells, though developed with some latency.
\end{abstract}

\begin{abstract}
...I made experiments on myself and my assistant, using smaller doses, and not repeating them so often...
\end{abstract}

- Clouston 1870

The biological actions of endogenous cannabinoids (eCB) occur by binding to the $\mathrm{CB}_{1}$ and $\mathrm{CB}_{2}$ receptors throughout the whole body (Ameri 1999; Pertwee 2006; Hill et al. 2007; Yoshida et al. 2011). The density of $\mathrm{CB}_{1}$ receptors in the amygdala is comparably high in mammals (Herkenham et al. 1990).

Amygdala similar to hippocampus is important for memory formation and often studied to elucidate plasticity at cellular level using the classical paradigm of Pavlov that continuously serves as a substrate (Pavlov 1927; Bliss and Lomo 1973; Rogan et al. 1997). The amygdala not only receives, but also sends behavior underlying signals into other regions (Racine et al. 1983; Aggleton and Mishkin 1984). While the role of hippocampus is crucial for memory formation, those associated with many different kinds of emotions are mainly modulated by the amygdala (Bucherelli et al. 2006; Fujii et al. 2020). The memory enabling substrate is a longterm potentiation (LTP) of neurotransmission into the postsynaptic neurons (Rogan et al. 1997; Kodirov et al. 2006).

The short-term synaptic plasticity in the form of depolarization-induced suppression of either excitation or inhibition (DSE and DSI) has been reported in several regions of the brain (Alger et al. 1996; Kano et al. 2009; Ivanova and Storozhuk 2011). We have discovered DSE in the lateral amygdala (LA), specifically at cortical inputs into the pyramidal neurons (Kodirov et al. 2010).

Despite the extensive studies on DSE and DSI, there are only three papers on interneurons that we are aware of. Two of them describe the presence of these phenomena: One study was carried out on parvalbumin immunoreactive interneurons of the stratum radiatum in the hippocampus (Ali 2007) and another on cerebellar stellate and basket cells (Beierlein and Regehr 2006). However, none of the cortical interneurons exhibited DSI despite the pres-

Corresponding author: skodirov@fm.ul.pt

Article is online at http://www.learnmem.org/cgi/doi/10.1101//m.052555.120. ence of a functional and cannabinoid-sensitive inhibitory inputs (Lemtiri-Chlieh and Levine 2007). The retrograde neurotransmission (Llano et al. 1991) takes place via the release of two natural ligands of endogenous cannabinoids anandamide and 2-arachidonoyl-glycerol (Urbanski et al. 2009). These ligands also suppress the evoked excitatory neurotransmission when applied exogenously in vitro (Ameri et al. 1999; Ameri and Simmet 2000; Lemak et al. 2007).

Since DSE in interneurons has not been reported in amygdala and we demonstrated the existence of DSE in pyramidal cells of LA (Kodirov et al. 2010), we then studied the same phenomenon in regard to interneurons; the main question was whether or not does depolarization-induced mobilization of eCBs from the two types of postsynaptic LA neurons cause similar retrograde modulation of cortical inputs? Subsequently, DSE between the presynaptic terminals and interneurons was shown, and we found that its properties are similar to those in pyramidal cells of LA. Our study documents the participation of endogenous cannabinoids of interneurons in DSE.

\section{Results}

The existence of depolarization-induced suppression of excitation (DSE) was proven in interneurons of the lateral amygdala (LA). The action potentials (APs) were evoked upon 1-sec depolarizing 100 pA currents, and based on their phenotype, the recorded neurons were identified either as interneurons (Fig. 1B) as compared with pyramidal cells (see Fig. 10A, below). Under these conditions, only a slight adaptation in both the peak amplitude and APD-action potential duration was observed (Fig. 1C). The

\footnotetext{
(C) 2021 Kodirov et al. This article is distributed exclusively by Cold Spring Harbor Laboratory Press for the first 12 months after the full-issue publication date (see http://learnmem.cshlp.org/site/misc/terms.xhtml). After 12 months, it is available under a Creative Commons License (AttributionNonCommercial 4.0 International), as described at http://creativecommons. org/licenses/by-nc/4.0/.
} 
A

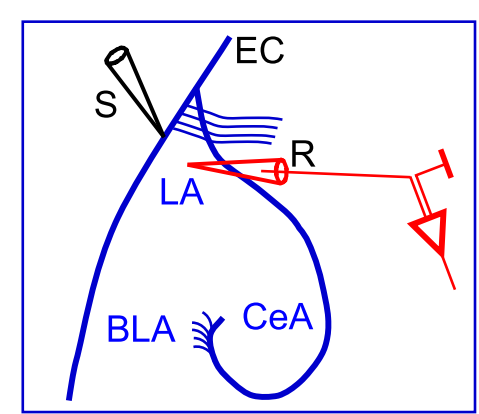

C

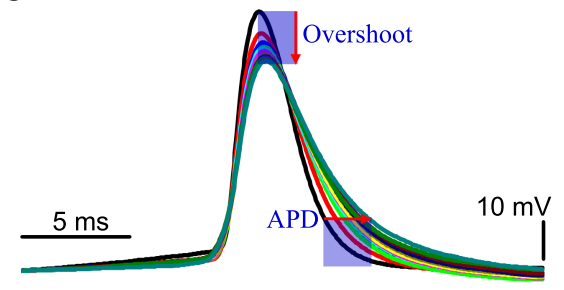

B

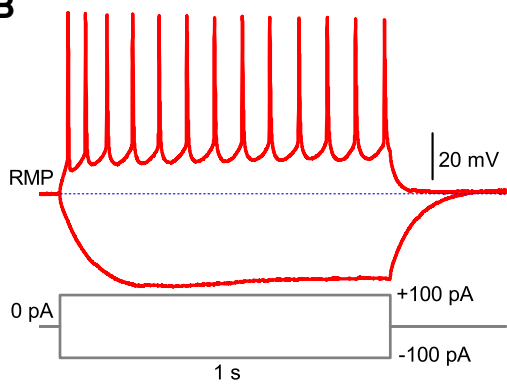

D
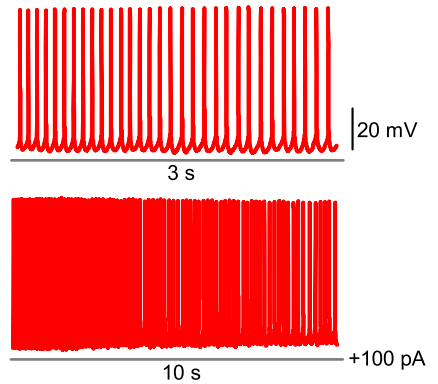

Figure 1. Experimental setup and action potentials phenotype. ( $A$ ) Schematic drawing of amygdala in coronal plane and the electrodes positions. (LA) Lateral nucleus of amygdala, (BLA) basolateral nucleus of amygdala, (CeA) central amygdaloidal nucleus, $(E C)$ external capsule, $(R)$ recording electrode, $(S)$ stimulating electrode. $(B, C)$ Electrophysiological properties of two representative interneurons in LA. (RMP) Resting membrane potential in $B:-65.5 \mathrm{mV}$; in C: $-60.1 \mathrm{mV}$. (D) Spontaneously generated spikes in LA interneuron. Note a small adaptation in rate during $3 \mathrm{sec}$, while RMP depolarization within $10 \mathrm{sec}$. Amplitude and time scale bars are shown. The duration of pulse protocol identically reflects the time scale for $B$.

interneurons of the LA fire continuously with regular intervals during 3-sec (Fig. 1D) or even 10-sec depolarization of membrane potential (MP) induced by $100 \mathrm{pA}$. Note a small increase in steady-state MP values in-between APs toward the end of $10 \mathrm{sec}$ pulse accompanied by a decrease in frequency. These properties are clearly distinct from pyramidal neurons under identical conditions, that is, they show faster adaptation within $1 \mathrm{sec}$ and fire often only up to four spikes around rheobase (Kodirov et al. 2010). In order to avoid forcing interneurons to fire excessively, only a minimal current injection was applied that did not exceed the 100-pA level. The mean resting membrane potential (RMP) of recorded cells was $-60.7 \pm 1.6 \mathrm{mV}(n=34)$.

\section{Retrograde neurotransmission in the LA}

The spontaneous excitatory postsynaptic currents (sEPSCs) were recorded at a HP-holding potential of $-70 \mathrm{mV}$ before and after the depolarization of MP to $\pm 0 \mathrm{mV}$ (Fig. 2A). All recordings were performed in the presence of PTX. As illustrated in Figure 2B, experiments and subsequent analyses were performed only on interneurons exhibiting sEPSCs with moderate frequencies and clearly distinguishable (from the baseline noise) amplitudes. Application of transient voltage pulse resulted in DSE as shown with 2-sec traces in Figure 2B in an expanded time scale immediately before and after depolarizing step. However, for cumulative probabilities longer traces were analyzed, and at most, $60 \mathrm{sec}$ depending on the overall release frequencies. Note that analyzed and compared durations were identical for paired conditions, that is, before and after the depolarization. The level of baseline membrane noise was on average $\pm 2 \mathrm{pA}$ and did not alter after depolarization as estimated in both Clampfit and Mini analyses programs (see also Fig. 2B). Thus, there was a minimal change in patch quality under these experimental conditions. Nevertheless, each event was examined manually in order to avoid including any artifacts. The depolariz- ing paradigm significantly affected only cumulative probability of inter-event intervals $(P<0.03$, Kolmogorov-Smirnov test) (Fig. 2C) but not the amplitude ( $P=$ 0.5) (Fig. 2D).

The DSE induction decreased the numbers of sEPSCs (190, bin size $2 \mathrm{pA})$ compared with those 241 during the baseline with corresponding mean values of $144 \pm 72$ versus $201 \pm 90 \quad(n=7, P<$ 0.02 ). The overall frequency of sEPSCs also significantly decreased from $6.2 \pm$ 1.7 to $4.4 \pm 1.3 \mathrm{~Hz}(n=7$, [*] $P<0.01)$ (Fig. $3 \mathrm{~A})$. Unexpectedly, the mean value of amplitudes of all events analyzed for the identical periods was greater after the depolarization $(7.12 \pm 0.68$ vs. $8.07 \pm 0.83$ pA, $n=7$, $\left.\left.{ }^{*}\right] P<0.01\right)$ (Fig. 3A). These effects persisted during $20 \mathrm{sec}$ (Fig. 3B,C). Also the median values have increased $(6.5 \pm 0.7$ vs. $5.8 \pm 0.6 \mathrm{pA}, n=7, P=0.02)$. Further analyses of events with either minimal $(2.7 \pm 0.2$ vs. $3.2 \pm 0.4 \mathrm{pA}, n=7$, $P>0.05)$ or maximal amplitude $(25.1 \pm$ 4.6 vs. $25.9 \pm 3.1 \mathrm{pA}, n=7, P=0.7)$ did not reveal significant differences.

\section{DSE at cortical inputs}

In order to examine the effects of postsynaptic depolarization on cortical terminals, we have evoked the EPSCs by stimulating the external capsule (Fig. 1A). In this set of experiments, the baseline amplitude of eEPSCs and those after the depolarization were compared at $-70 \mathrm{mV}$ (Fig. 4A,D). We have observed the significant latency preceding the suppression of eEPSCs, that is,

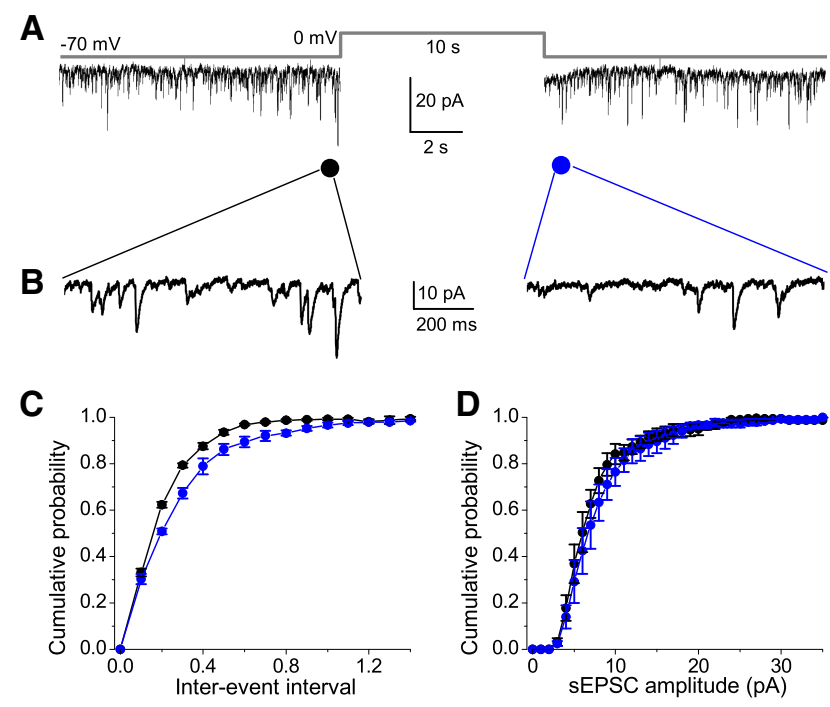

Figure 2. The DSE of spontaneous EPSCs in interneurons of the LA. $(A)$ sEPSCs were recorded at holding potential of $-70 \mathrm{mV}$; test potential was $\pm 0 \mathrm{mV}$. (B) sEPSC pattern immediately before and after the 10-sec depolarizing step in an expanded time scale. Identical scale bars refer to both traces in $A$ and $B$. Black and blue symbols are sEPSCs amplitude/rate immediately before and after the depolarizing pulse, respectively. $(C, D)$ Cumulative frequency $(P<0.03$, Kolmogorov-Smirnov test) and amplitude $(P>0.05)$ probabilities, respectively. Error bars are \pm SEM. 
A
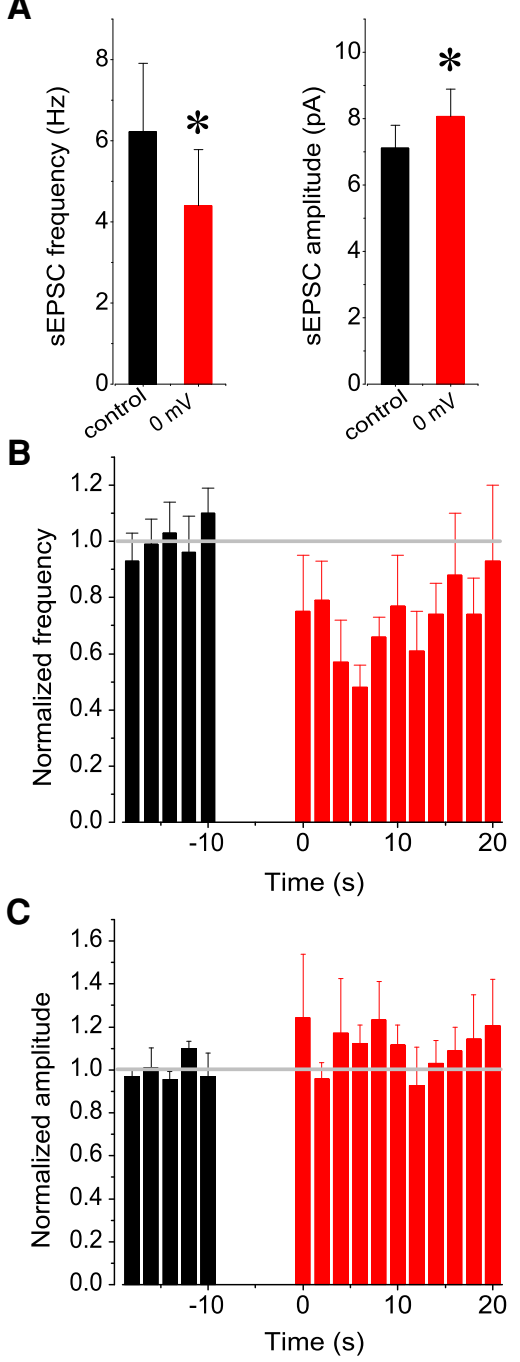

Figure 3. Frequency and amplitude patterns during DSE in interneuron. (A) Mean frequency (left panel, $\left.n=7,{ }^{*}\right] P<0.01$, paired $t$-test) and amplitude (right, $\left[{ }^{*}\right] P<0.02$ ) of spontaneous EPSCs. Note a significant decrease in frequency, but an increase in amplitude (see Results). $(B, C)$ The time course of normalized frequency and amplitude values (bin size $2 \mathrm{sec})$.

DSE, upon stimulation of cortical inputs (Fig. 4B,C). The DSE was present in 11 out of 16 interneurons with a maximal value of $72 \% \pm 3 \%$ of the baseline $\left(\left[{ }^{*}\right] P=0.001\right.$ ) (Fig. $4 \mathrm{~F}$ ); in remaining cells, the depolarization did not affect the amplitude of eEPSCs. Figure $4 \mathrm{~F}$ also summarizes results obtained from a subpopulation of neurons, which similar to some of pyramidal cells did not exhibit DSE (99\% $\pm 1 \%$ of baseline, $n=5, P=0.1$ ). Since the electrophysiological parameters of these two groups of interneurons were similar (Table 1 ), either the presence or the absence of DSE is not related to the passive and active properties of the studied cells. However, the results revealed that the depolarization-induced suppression at excitatory inputs into the interneurons of the LA (Fig. 4C,F) was comparable with pyramidal cells (see Fig. 10, below). Moreover, the suppression in interneurons persisted during an identical time frame. Therefore, in some cells, we followed the magnitude of the DSE over an extended period and found that the suppression was reversible within $4 \mathrm{~min}$ (Fig. 5A); this was also true for the neuron shown in Figure 4, A and B. The Gaussian fit to data points revealed the DSE peak at $0.94 \mathrm{~min}$ and, thereafter, a slow recovery toward the baseline values. The mean values from three cells confirmed the peak DSE $(72 \% \pm 6 \%$ of baseline, $P=0.04)$ and recovery timings in interneurons of the LA $(104 \% \pm 13 \%, P=0.7$, Fig. 5B).

\section{Role of endogenous cannabinoids in retrograde neurotransmission in LA}

The two major contra-balancing excitatory and inhibitory neurotransmissions in the brain are prone to DSI and DSE, which is a result of activity in the endogenous cannabinoids loop. Nonetheless, we have experimented whether or not the DSE in interneurons of LA are comparable in this regard. After revealing the presence of DSE in certain interneurons, they were exposed to 3 or $10 \mu \mathrm{M}$ AM-251 (Fig. 4A-C). The inhibition of presynaptic $\mathrm{CB}_{1} \mathrm{R}$ by this selective antagonist prevented the DSE (Fig. 4F). The ratio of amplitude was estimated under these conditions, which revealed the mean value of $96 \% \pm 1 \%$ of baseline $(n=3, P>0.05)$ after the depolarization. Thus, despite the differences in overall magnitudes of DSE in pyramidal cells (Kodirov et al. 2010) and interneurons (Fig. 4C,F), the suppression was comparably prevented by AM-251.

Moreover, values from the eight out of 11 interneurons susceptible to DSE revealed the transient increase in eEPSC (Fig. 6D). Therefore, we have reanalyzed responses of pyramidal cells (Kodirov et al. 2010) and revealed a similar tendency (Fig. 6A,B). Although the amplitude of the first EPSC (16th sweep) (Fig. 6C) recorded after the depolarizing step is significantly higher $(148 \% \pm$ $7 \%$ of baseline, $n=6, P<0.001$ ), the underlying mechanism might be related to the intrinsic properties of the postsynaptic neurons, but not to depolarizing step. Alternatively, the presynaptic terminals sense the changes in inter-stimulus intervals. The neuron simply shows a rapid adaptation to a new condition, which in this case is a 10- versus 3-sec interval. This view is consistent with our results under baseline conditions, since the normalized amplitude of the first sweep is greater than that of the second one in pyramidal cells $(120.8 \pm 7.0$ vs. $92.4 \% \pm 3.9 \%, n=6, P<0.001)$ (Fig. 6 C). Thus, this observation reveals that the synapse between the cortical terminal and this particular neuron did not experience a higher stimulation rate (at $3 \mathrm{sec}$ interval) prior to the very start of baseline recordings. In interneurons, this phenomenon was less pronounced and the mean normalized amplitude values of the first and second sweeps were not significant ( $111 \pm 5$ vs. $99 \% \pm 8 \%$ of baseline; $n=8, P=0.2)$ (Fig. 6D). Nevertheless, the amplitude of the 16th EPSCs in interneurons was also increased to $120 \% \pm 5 \%$ of baseline $(n=8, P<$ $0.01)$. Since the latter value is significantly smaller than those observed in pyramidal cells (see above), it reveals the additional (to firing properties) differences among the two types of neurons in regard to this phenomenon. Also, a distinctive feature of DSE in both neuronal types emerges during the representation mentioned earlier (Fig. 5). These are their magnitudes and latencies.

Table 1. The electrophysiological parameters of two groups of interneurons in the LA

\begin{tabular}{lcc}
\hline LA interneurons & \multicolumn{2}{c}{ DSE } \\
\hline Parameters & Absent & Present \\
\hline AP amplitude $(\mathrm{mV})$ & $77.6 \pm 7.4$ & $81.8 \pm 4.3$ \\
AP numbers $(\mathrm{at} 100 \mathrm{pA})$ & $13.8 \pm 3.2$ & $10.8 \pm 0.7$ \\
AP threshold $(\mathrm{mV})$ & $-30.8 \pm 2.6$ & $-36.3 \pm 2.3$ \\
Input resistance $(\mathrm{M} \Omega)$ & $391.9 \pm 54.4$ & $369.4 \pm 34.0$ \\
Overshoot $(\mathrm{mV})$ & $23.2 \pm 6.6$ & $21.8 \pm 4.2$ \\
RMP (mV) & $-54.3 \pm 3.4$ & $-60.0 \pm 2.3$ \\
$n$ & 5 & 11
\end{tabular}


A
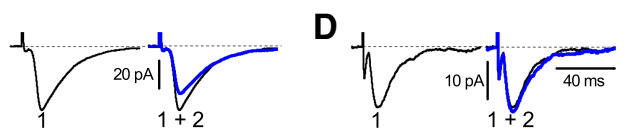

B

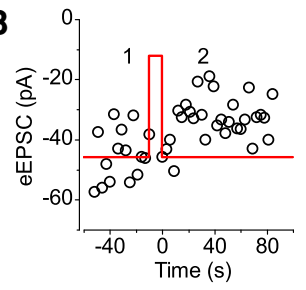

C

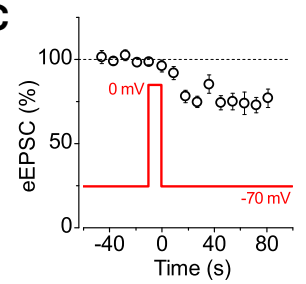

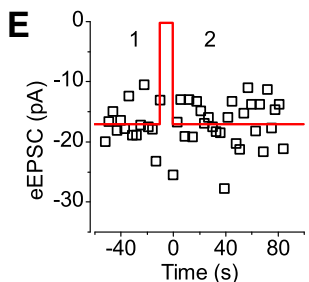

$\mathbf{F}$

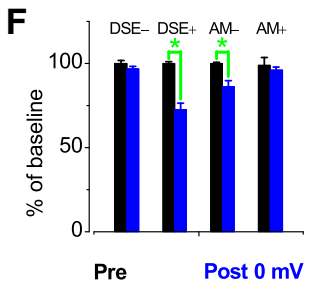

Figure 4. Depolarization-induced suppression of evoked EPSCs in interneurons of LA. ( $A$ ) The superimposed traces of eEPSCs before (left panel) and after the test pulse $(0 \mathrm{mV}$; right). (B) Data points collected at a holding potential of $-70 \mathrm{mV}$ during the same experiment shown in $A$. (C) Normalized values $(n=11)$. $(D, E)$ Response of interneuron that did not exhibit DSE. $(F)$ Summary of data revealing the absence $(n=5)$ and presence $\left(n=11,\left[^{*}\right] P<0.001\right)$ of DSE in subpopulations of interneurons. When either 3 or $10 \mu \mathrm{M} \mathrm{AM-251}$ was applied to same neurons, the DSE was blocked by $\left.\left(n=3,{ }^{*}\right] P=0.026\right)$. Values before and after the 10 -sec step for each condition are color matched as Pre and Post $0 \mathrm{mV}$. Identical scale bars for $A$ and $D$ are shown and error bars in $C$ and $F$ are \pm SEM.

As it can be estimated from Figure 6, C and D, the mean latency of $18 \mathrm{~s}$ in interneurons was two times longer than that of pyramidal cells.

\section{Postsynaptic neuron's $\mathrm{Ca}^{2+}$ content and presynaptic neurotransmitter release}

We have compared the magnitude of DSE under two experimental conditions concerning the intracellular $\mathrm{Ca}^{2+}$ contents. This was achieved by using two different concentrations of EGTA, which was set at 0.2 and $10 \mathrm{mM}$. The low concentration was used for main body of study, while the high content of EGTA for chelation purpose. Since the DSE is not present in all cells, its absence in presence of $10 \mathrm{mM}$ EGTA (Fig. 7) difficult to ascertain. However, in exemplified interneuron, the DSE paradigm exerted and influenced the response. That is the amplitude diapasons were narrow during prestimulus (gray box), while increased after (green, Fig. 7B). When compared with mean of 15 baseline amplitudes (red dashed line), there was an increase after the step during equal time. A high EGTA and either cortical stimulation or postsynaptic depolarization did not influence the dynamics and phenotype of spontaneous EPSCs during $600 \mathrm{msec}$ (Fig. 7A, asterisk). Finally, there was a slight and insignificant increase in amplitudes of eEPSC that serves as positive control (Fig. 7B).

Furthermore, in this context we also analyzed the coefficient of variation $\left(\mathrm{CV}^{2}\right)$ of the amplitude of eEPSC (Faber and Korn 1991; Alger et al. 1996) in individual interneurons within these two groups. The ratios of DSE in individual cells were plotted as abscissa values and those of $\mathrm{CV}^{2}$ as ordinate in order to validate either the presynaptic or postsynaptic origin of DSE (pre and post) (Fig. 8).

The chelation of $\mathrm{Ca}^{2+}$ content in postsynaptic cells diminished the DSE in five out of six interneurons tested; the $\mathrm{CV}^{2}$ ratio

decreased only in two cells (Fig. 8A). Thus, under these conditions, the amplitude of eEPSCs, despite the depolarization, remained unaffected with a mean value of $101 \% \pm 4 \%$ of the baseline, $(n=6, P=$ $0.9)$. Also, the corresponding average value of the $\mathrm{CV}^{2}$ ratio $(95 \% \pm$ $5 \%$ ) was not significantly apart compared with the baseline; the latter two values are represented with the single green filled circle and the respective SEM are considered.

The depolarizing step applied to those interneurons dialyzed with 0.2 mM EGTA (Fig. 8B) significantly suppressed the amplitude of eEPSCs $(74.5 \% \pm 3.7 \%$ of baseline). Note that the latter value is an average of 15 responses and hence slightly differs from the maximal suppression presented in Figure 4F. This procedure is required for the calculation of the corresponding $\mathrm{CV}^{2}$ before and after the depolarization. The DSE was accompanied with a decrease in the $\mathrm{CV}^{2}$ ratio (blue empty circle) to $74.6 \% \pm 9.8 \%$ of the baseline (green filled circle, $n=11, P<0.001$ ). However, note that only in one interneuron (out of 11 totals) the $\mathrm{CV}^{2}$ ratio was significantly increased (red empty diamond). Despite the inclusion of this outsider to presented mean value (green filled circle), a prevailing presynaptic site of effects was observed. Collectively, the DSE occurs only at physiological $\mathrm{Ca}^{2+}$ concentration (Fig. 4), and it is a result of transient decrease in glutamate release (Fig. 8B; see also Fig. 3).

\section{Absence of presynaptically evoked suppression of excitation}

The suppression of neurotransmission can be achieved not only by depolarization of the postsynaptic neuron, but also by brief trains of action potentials evoked by stimulation of innervating fibers (Brown et al. 2003). This phenomenon is known as synaptically evoked suppression of excitation (SSE), which involves the mGluR1-mediated release of endocannabinoids. We have performed similar experiments at neurons' resting membrane potentials in current-clamp mode. The amygdalar neurons are vulnerable to LTP induced by very moderate stimuli in vitro. The induction paradigm consists of presynaptic stimulations at $2 \mathrm{~Hz}$ paired with $40 \mathrm{sec}$ depolarization/APs (see Kodirov et al. 2006).
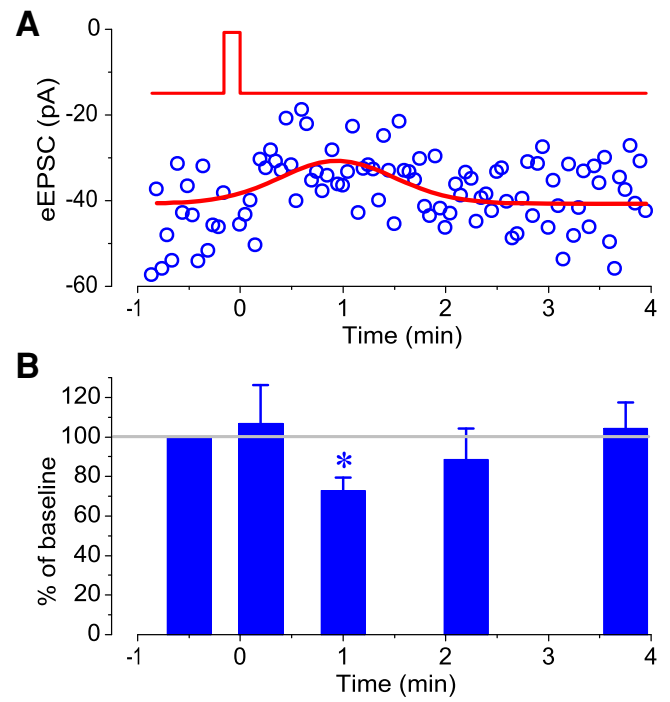

Figure 5. Reversibility of DSE with the time. $(A)$ The amplitude dynamic of evoked EPSCs within 4 min after the depolarization in the same neuron shown in Figure 4, A and B. The entire experimental amplitude data points were fitted with Gaussian function yielding the peak of DSE at $0.94 \mathrm{~min}$. $(B)$ The normalized mean values for three cells at indicated time points before and after the depolarization. The identical holding and test potentials were used. 
A

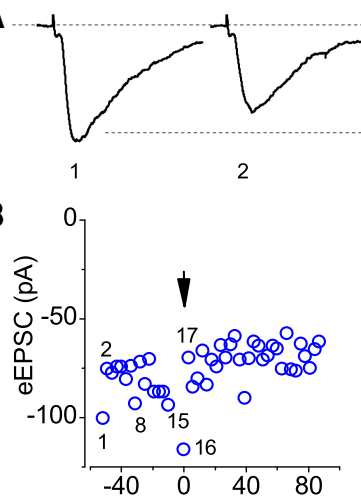

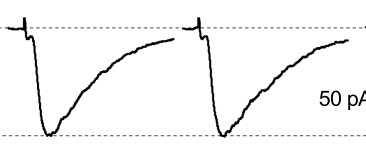

8

15

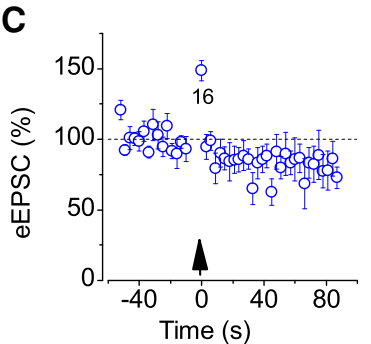

D

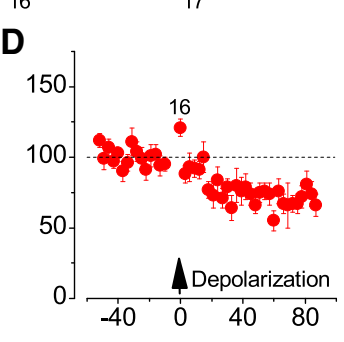

Figure 6. Transient increase in glutamatergic neurotransmission induced by brief depolarization of postsynaptic neurons. ( $A$ ) Evoked EPSCs at different time points indicated by numbers with corresponding sweeps; 1 and 17 refer to the first and 17th sweeps, respectively. Note the 10-sec depolarization between 15th and 16th sweeps; otherwise inter event interval is $3 \mathrm{sec}$. (B) Amplitude values from the single experiment at a holding potential of $-70 \mathrm{mV}$; representative traces are shown in $A$. $(C, D)$ Corresponding relative mean values of data points for pyramidal cells ( 16 th: $148.8 \% \pm 7.2 \%$ of baseline, $n=6, P<0.001)$ and interneurons $(120.6 \% \pm 5.9 \%$ of baseline, $n=8, P<0.01)$; the amplitude and time scales are identical for all traces and error bars are \pm SEM.

Although, this experimental paradigm is weak compared to those used for other brain regions (Fujii et al. 2016), we have avoided a similar approach (i.e., no APs were evoked during the stimulation). Instead, we have stimulated cortical inputs into the interneurons at a logarithmic 1,10 , and $100 \mathrm{~Hz}$ (a train consisted of 10 consecutive stimuli each) (Fig. 9A,D,G). As it is evident from Figure 9, G-I, even this paradigm at $100 \mathrm{~Hz}$ led to a potentiation of glutamate release in interneurons. Under these conditions, only the first response immediately after the train was dramatically suppressed resembling a synaptic failure achieved during a minimal stimulation paradigm (S Kodirov, unpubl.). At $100 \mathrm{~Hz}$ the mean value of the first responses was $53 \% \pm 12 \%$ of baseline $(n=9, P<0.01)$ (Fig. 9I). Only in one out of these nine cells the potentiation of the first response immediately after the stimulation occurred. At $10 \mathrm{~Hz}$, similar tendencies were observed albeit the lower magnitude $(69 \% \pm 7 \%$ of the baseline, $n=11, P<0.01)$ (Fig. 9F). Although at $1 \mathrm{~Hz}$ an average response was smaller under these experimental conditions, it was not significant $(83 \% \pm 10 \%$ of baseline, $n=12, P=0.1$ ) (Fig. 9C). However, during the delivery of stimuli (data not shown), the glutamate release was temporarily decreased, similar to the evoked inhibitory neurotransmission (Kodirov et al. 2006). In Figure 9, C, F, and I, for clarity, each three data points are averaged except for the magnitude of the first, second, and third sweeps immediately after the stimulation. Collectively, in the lateral amygdala, under our experimental conditions, the SSE was absent.

\section{Similitude of DSE in interneurons and pyramidal cells}

For comparison purpose the DSE of evoked EPSC in pyramidal neurons were registered (Fig. 10). While the majority of neurons of this type trigger up to five rate adaptation prone APs during $1 \mathrm{sec}$ (Kodirov et al. 2006, 2010), there are those exhibiting a solitary spike upon marginal +50 pA current injections (Fig. 10A). Note that subsequent steps applied at 10 or $1 \mathrm{~Hz}$ did not excite the cell. Moreover, the magnitudes of MP depolarization during repeated steps were comparable or even gradually decreased. The RMP at the beginning and the end of this 12 sec experiment remained similar at $-58 \mathrm{mV}$. The same applies as to a slow depolarization upon the onset of the step, latency, threshold, and

amplitude of two spikes. The magnitude of mean-normalized DSE was $84 \% \pm 1 \%$ $\left(n=6, \quad\left[{ }^{*}\right] \quad P<0.04\right) \quad$ (Fig. $\left.\quad 10 \mathrm{~B}-\mathrm{E}\right)$. Although, the latter value is different from that estimated for interneurons $(72 \% \pm 3 \%$ of the baseline, $n=11$ ) (Fig. $4 \mathrm{~F})$, the comparison did not reveal statistical power $(P=0.08)$. Moreover, in contrast to previous study (Kodirov et al. 2010), a half of tested cells did not exhibit DSE. Corresponding age and RMP of these two groups of mice and cells were not far apart (DSE ${ }^{+}, \mathrm{P} 31 \pm 1$ and $-57 \pm 2$ $\mathrm{mV}$ vs. $\mathrm{DSE}^{-}, \mathrm{P} 29 \pm 1$, and $\left.-65 \pm 4 \mathrm{mV}\right)$.

\section{Discussion}

Despite the advances in research addressing a subtype of the short-term synaptic plasticity known as DSE or DSI (Kano et al. 2009), there are only few papers describing these phenomena in amygdala: two in the BLA (Zhu and Lovinger 2005; Patel et al. 2009) and one in the LA (Kodirov et al. 2010). The first two papers describe DSI and deal only with local networks involved in eCB signaling. Therefore, the expression of $\mathrm{CB}_{1} \mathrm{Rs}$ in terminals of either cortical or thalamic inputs into the BLA, LA, and CeA remained suggestive.

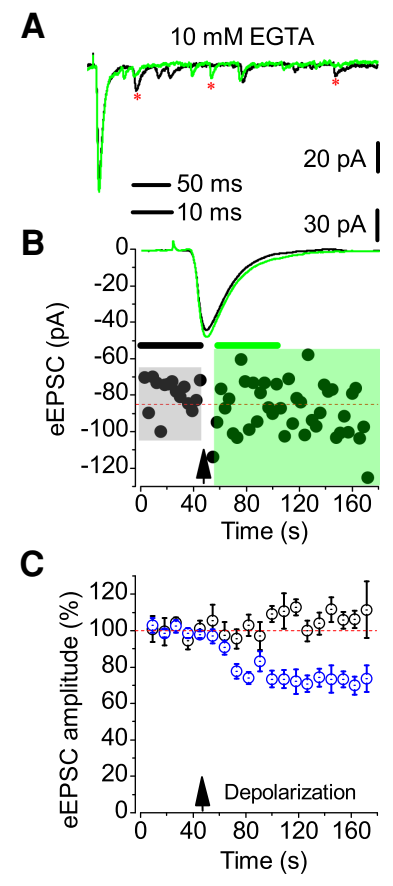

Figure 7. Absence of DSE in the presence of high EGTA. (A) Identical paradigm as in Figure 4 was used to trigger depolarization-induced suppression of evoked EPSC in interneurons of LA. Two representative traces closely resembling the average of 15 responses as in $B$ are shown. Note the comparable presence of spontaneous EPSCs before ( ${ }^{*}$, black) and after the $\pm 0 \mathrm{mV}$ pulse (green). (B) Amplitudes at a holding potential of $-70 \mathrm{mV}$ for both conditions. (C) Response of interneurons in $10(n=6$, black) and $0.2 \mathrm{mM}$ intracellular EGTA $(n=11$, blue, same data as in Fig. 4C). 
A

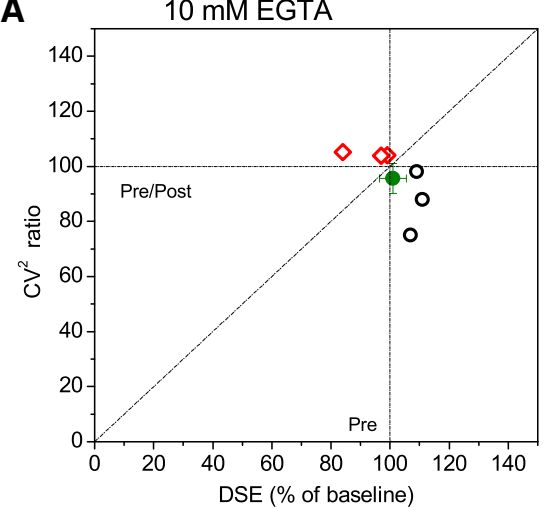

C

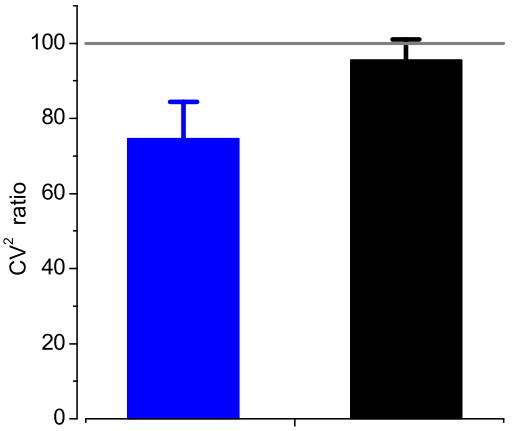

0.2
B

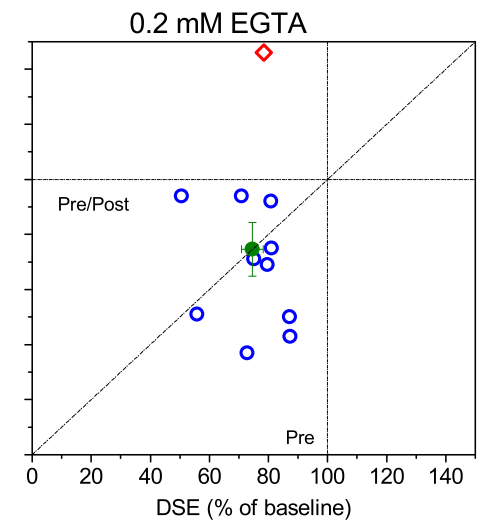

D

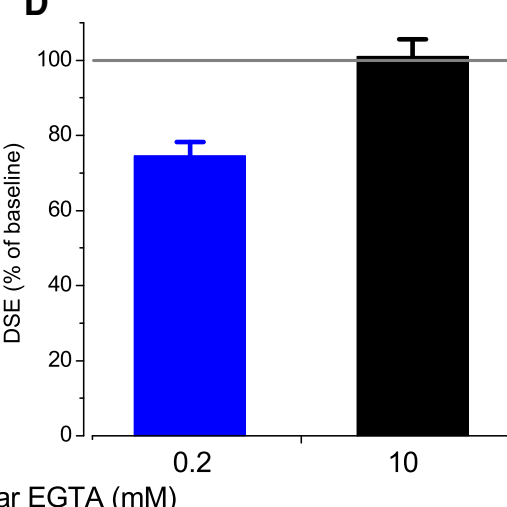

Figure 8. The $\mathrm{Ca}^{2+}$ influx dependence of DSE and its presynaptic site of effects in the LA. $(A, B)$ Percentage of DSE and coefficient of variation $\left(C^{2}\right)$ in individual interneurons analyzed using $10 \mathrm{mM}$ EGTA $(n=6)$ and $0.2(n=11)$, respectively. The individual DSE and a decrease in $\mathrm{CV}^{2}$ ratio are shown (blue empty circle). Note a significant increase (red empty diamond) in $\mathrm{CV}^{2}$ ratio in one of interneurons. Mean DSE and $\mathrm{CV}^{2}$ ratio are shown with a single symbol (green filled circle) revealing the absence of DSE during intracellular $\mathrm{Ca}^{2+}$ chelation. Data are derived from experiments similar to Figures 4-7. The mean \pm SEM bars are shown for both $x$ and $y$ directions. $(C, D)$ Direct comparison of two conditions revealing a correlation between DSE and reduction in $\mathrm{CV}^{2}$ ratio. The $\mathrm{CV}^{2}$ ratio is derived form $\mathrm{CV}^{2}$ pre- $/ \mathrm{CV}^{2}$ postdepolarizing step.

We have reported DSE at synapses between the cortical inputs and pyramidal neurons of the LA (Kodirov et al. 2010). The depolarization of postsynaptic cells during the $5 \mathrm{sec}$ is considered and established to be physiological. However, by comparing 5 versus $10 \mathrm{sec}$ we did not observe any differences (data not shown). An even shorter duration should lead to the release of endocannabinoids, that is, sn-2 arachidonylglycerol (2-AG) and anandamide (Devane and Axelrod 1994), and subsequently transiently activate $\mathrm{CB}_{1}$ Rs. The level of natural ligand 2-AG in BLA is elevated under repeated psychological stress, which increases both the magnitude and duration of DSI (Patel et al. 2009). However, such an elevation is not transient in terms of DSI kinetics mediated by eCB. It is well documented that the tonic release of endogenous ligands is absent in the brain (Lemak et al. 2007; Urbanski et al. 2009, Kodirov et al. 2010). Therefore, the relationship between the stress and depolarization induced 2-AG elevation/release in BLA is not entirely clear. Could depolarization induced eCB release be additive to those occurring under stress? Interestingly, acute stress decreased the anandamide level in amygdala (Hill et al. 2009), while those of 2-AG did not significantly change (Patel et al. 2009). Nevertheless, in the latter two studies the 2-AG levels within the amygdala slightly decreased. These outcomes support the role of endogenous cannabinoids in multiple pathological states of human brain (Melamede 2009).
The depolarization of MP in interneurons suppressed the spontaneous EPSCs derived mostly by local neuronal network in the LA (Fig. 2). The proportion of interneurons exhibiting DSE was comparable with those of pyramidal cells (Kodirov et al. 2010). The DSE was also observed during recordings of evoked EPSCs (Fig. 4), which were enabled by the stimulation of cortical inputs into the LA interneurons; the latter input is a crucial part of fear circuits. The retrograde suppression involves the intracellular $\mathrm{Ca}^{2+}$ increase in general, which can be proven by inclusion of either BAPTA or higher concentration of EGTA in the pipette solution (Figs. $7,8)$. The overall features of DSE in interneurons in this regard were also similar to pyramidal cells (Kodirov et al. 2010).

A striking difference in interneurons compared with pyramidal cells was an increase observed in average amplitude values of sEPSCs immediately after the depolarization. Further event distribution analyzes revealed that this resulted by differential effects of DSE on certain range of sEPSCs amplitudes and was a transient phenomenon similar to those described for the amplitude of eEPSCs. For example, in these experiments the distributions of event numbers with the amplitude of 10 pA were increased (Fig. 3F). Similar to pyramidal cells (Kodirov et al. 2010), the depolarization did not suppress the frequency and amplitude of mEPSCs in interneurons (data not shown).

However, the frequencies of sEPSCs were suppressed by the depolarization, which proves the presynaptic origin of the DSE. Consistently, by analyzing and comparing the magnitude of DSE and respective $\mathrm{CV}^{2}$ (Faber and Korn 1991), we have defined the presynaptic site as a target for endogenous cannabinoids released from interneurons during the activation (Alger et al. 1996; Kodirov et al. 2010).

The DSE in interneurons similar to pyramidal cells occurred with some latency after the depolarization (Kodirov et al. 2010). In some cells we observed even a transient $(<3 \mathrm{sec})$ increase in the amplitude of evoked EPSC. A similar latent period (1.2 sec) preceding the actual DSI was also shown in pyramidal neurons of the CA1 area of the hippocampus (Wilson et al. 2001). Therefore, a first data point (mean amplitudes of the first three eEPSC) obtained immediately upon termination of pulse sometimes did not show any suppression.

In the presence of a selective $\mathrm{CB}_{1}$ Rs antagonist, $\mathrm{AM}-251$, no suppression was observed under an identical induction paradigm in interneurons of the LA. Another similarity in DSE properties in these neurons was a transient increase in the amplitude of the first EPSC immediately after the depolarizing step. Such complex responses have been documented for the cerebellar interneuronPurkinje cell synapses (Duguid and Smart 2004). However, under these conditions, the depolarization resulted first in DSI that gradually decreased in magnitude and transformed into a depolarization-induced potentiation of inhibition (DPI) characterized by rebound increase in both the frequencies and amplitudes of miniature IPSCs. The retrograde communication has also been 
A

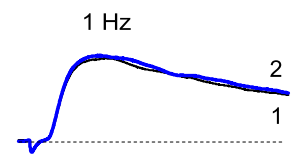

B

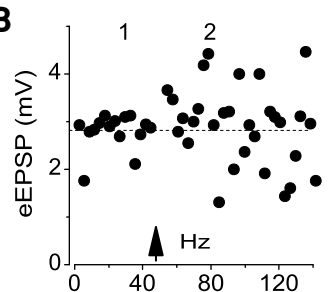

C

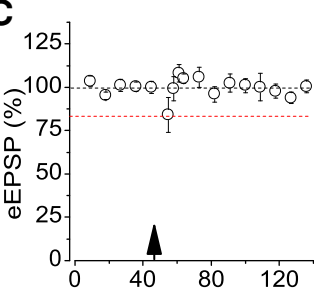

D

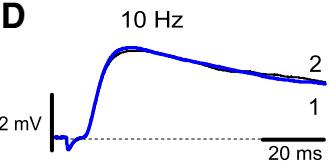

E

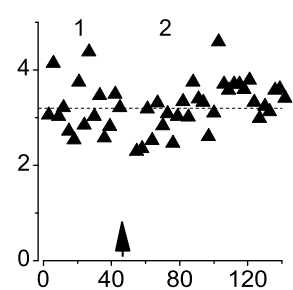

F

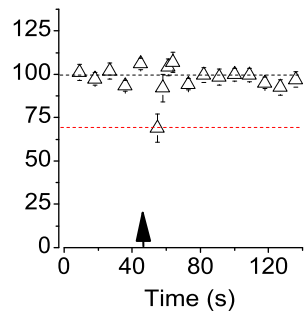

G

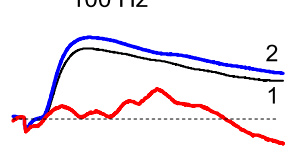

H

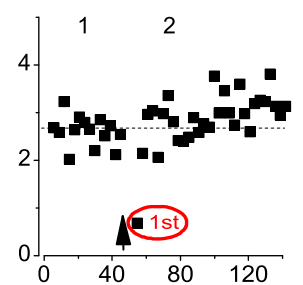

I

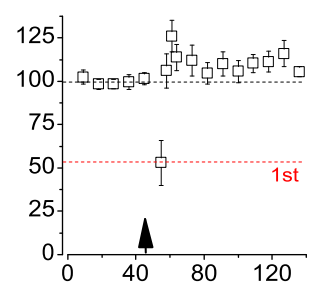

Figure 9. Effects of cortical inputs' stimulation on response of interneurons. $(A)$ The magnitude of eEPSPs before (1) and after (2) the application of $1 \mathrm{~Hz}$ paradigm are superimposed. (B) Data points collected at $0.33 \mathrm{~Hz}$ from the same single representative experiment shown in $A$. (C) Normalized mean amplitude values $(n=12)$. $(D-F)$ Recordings at $10 \mathrm{~Hz}(n=11) .(G-I)$ The response at $100 \mathrm{~Hz}(n=9)$; note almost complete absence of the first response to stimulation, however, spontaneously occurring EPSPs (red trace). Scale bars for $A, D$, and $G$ are identical. Error bars are \pm SEM.

animals were not disturbed unnecessarily. Only a single mouse per day was received from the staff immediately before preparation. Animals were always subjected to anesthesia and the handling protocol of the local committee was strictly followed.

\section{Brain slices}

The brains of 3- to 5-wk-old C57BL/6N female and male mice (P31.1 $\pm 0.9, n=22$ for interneuron experiments, while for principal cells, P30.8 $\pm 0.94, n=10$ ) were sectioned at coronal plane $(300 \mu \mathrm{m})$. Immediately after opening the skull a small portion of ice cold artificial cerebrospinal fluid (ACSF) was introduced to the exposed surface of brain in order to cool and easy the dissection. The isolated brain and slices were kept in ACSF.

\section{Patch-clamp}

All experiments were performed at room temperature $\left(\sim 24^{\circ} \mathrm{C}\right)$. The whole-cell configuration of the patch-clamp technique (Hamill et al. 1981; Krishtal 2015) was used in order to record the voltage and currents in neurons of the LA (Fig. 1A). The pipette resistance was in a diapason of 3-6 M $\Omega$; uncompensated series $(<40$ $\mathrm{M} \Omega)$ and input resistance $(375.3 \pm 18.1$ shown between monosynaptically connected hippocampal interneurons and pyramidal cells in culture. The evoked AP in the presynaptic neuron elicits an EPSC in the postsynaptic cell, while the depolarizing pulses in the latter neuron decreases the magnitude of this event. This phenomenon was termed "depolarization-induced NMDA receptor inactivation" (Medina et al. 1999), which consistently occurred when rather multiple than a single depolarizing step were used.

In conclusion, for the first time we described the DSE in interneurons of the LA. Next, the question as to whether or not the $\mathrm{CB}_{1}$ receptors of cortical inputs differentially respond to the mobilization of eCBs depending on neuronal subtypes at postsynaptic sites is answered (Fig. 11). The extent of suppression and its duration in interneurons was grater than in pyramidal cells. Since the latter recovered within $4 \mathrm{~min}$, this phenomenon is a DSE, but not depolarization induced long-term depression (LTD). The DSE in the LA was abolished by chelating the intracellular $\mathrm{Ca}^{2+}$ in the postsynaptic cell. In the absence of this key trigger, the endogenous cannabinoids are not released and subsequently the $\mathrm{CB}_{1}$ Rs are not activated in the presynaptic terminals. The type of short-term synaptic plasticity reported in this study could have an impact on fear memory by contributing to a dynamic fine balance of excitation and inhibition within the cortical inputs.

\section{Materials and Methods}

Mice were housed with same sex littermates and kept under most appropriate conditions with food and water being available ad libitum. They were exposed to simulated diurnal cycle and have also received environmental enrichments to explore and play. Furthermore, shelters in form of small paper boxes within the home cages were included to hide and sleep in. Most importantly, only dedicated and animal loving staff had an access to rooms, so
A

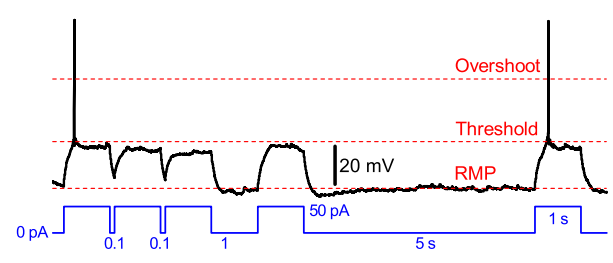

B

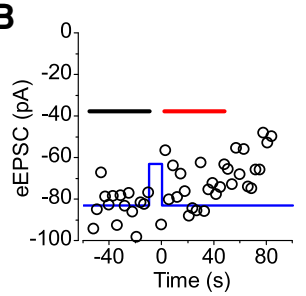

D

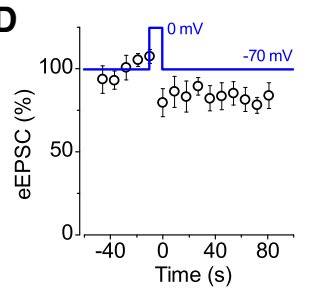

C

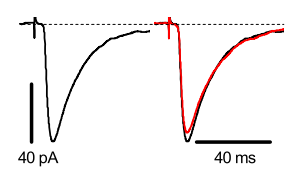

E

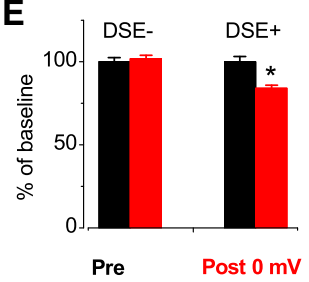

Figure 10. Depolarization-induced suppression of evoked EPSC in principal cells of LA. (A) Pyramidal neuron does not excite recurrently in response to rheobase current. The refractory period was at least $5 \mathrm{sec}$. Note the similitude in behavior of MP during two spikes. (B) The eEPSC in the same neuron immediately before and after $\pm 0 \mathrm{mV}$ step. Holding potential was $-70 \mathrm{mV}$. (C) Corresponding average traces of 15 eEPSCs before (black) and after the test pulse (red). ( $D$ ) Normalized values $(n=6)$. (E) The absence $\left(n=6, \mathrm{DSE}^{-}\right)$and presence $\left.\left(n=6,{ }^{*}\right] P<0.04, \mathrm{DSE}^{+}\right)$of suppression. Values before and after the 10 sec steps for each condition are color matched as pre and post $0 \mathrm{mV}$. 


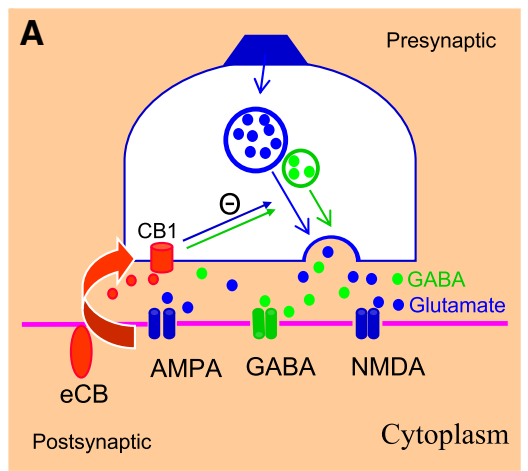

\section{B Afferent excitatory input}

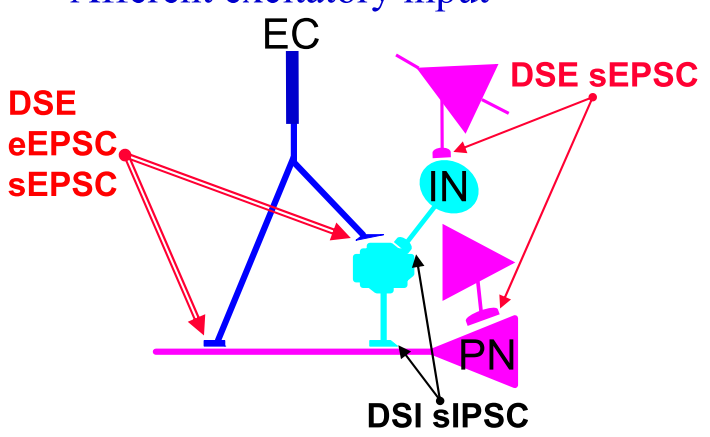

Figure 11. Retrograde communication among neurons and terminals within amygdala. $(A)$ The eCB cascade is similar whether the postsynaptic neuron receives cortical inputs or the neurotransmitter release from other cells. Modified (Kodirov et al. 2010). (B) Sites of DSE and DSI, types of ultrashort-term plasticity, in lateral amygdala. DSE of eEPSC refers to experimentally evoked glutamate release as the same may occur spontaneously (SEPSC). (DSE and DSI) Depolarization-induced suppression of excitation and inhibition, (EPSC and IPSC) excitatory and inhibitory postsynaptic currents, (IN) interneuron, (PN) principal or pyramidal neuron.

$\mathrm{M} \Omega, n=34$ ) were monitored during the experiments. The holding potential was set at $-70 \mathrm{mV}$ for the measurements of both spontaneous and evoked excitatory postsynaptic currents (EPSCs). The $\gamma$-aminobutyric acid receptor type $\mathrm{A}\left(\mathrm{GABA}_{\mathrm{A}}\right)$ activity was inhibited with $100 \mu \mathrm{M}$ picrotoxin (PTX). The EC was stimulated by either a bipolar tungsten electrode or sliver painted patch pipettes of lower resistance filled with equilibrated ACSF (Kodirov et al. 2010). The EPSCs were evoked by stimulation of cortical inputs at 0.33 $\mathrm{Hz}$ (100- to 150 - $\mu$ sec pulse width) before and after the depolarization of postsynaptic neuron. During the recording of unitary eEPSCs any failures were avoided. The action potentials (APs) were evoked in response to depolarizing current injection (up to $100 \mathrm{pA}$ ). The pyramidal cell and interneuron were identified by either frequency adaptation (Kodirov et al. 2010) or regular firing properties of APs (Fig. 1B).

A set of experiments in Figure 9 were conducted under current-clamp conditions, and this approach was chosen in order to investigate the neurons at their RMP and not to interfere with fine tuned physiological properties by introducing HP.

Clampex, Clampfit, Mini Analysis, Origin and Microsoft Excel software were used for data acquisition and subsequent analyses. The current signals were filtered at $1 \mathrm{kHz}$ and the sampling interval was 50-200 $\mu \mathrm{sec}$. The cumulative frequency (as in Fig. 2, bin size $10 \mathrm{msec}$ ) and amplitude (2-pA equal bins) graphs were constructed for corresponding data obtained under control conditions and upon termination of $10 \mathrm{sec}$ depolarizing step; the number of experiments $(n)$ and means \pm SEM are indicated. The automated Gaussian fit analysis was performed in Origin. In order to obtain the square root of the coefficient of variation $\left(\mathrm{CV}^{2}\right)$, first the $\mathrm{CV}$ was estimated as

$$
\mathrm{CV}=\frac{\mathrm{SD}}{\mathrm{MEAN}} \times 100 \%
$$

Where the SD is a standard deviation of 15 eEPSC amplitudes, MEAN-an average amplitude value of 15 eEPSCs. Identical parameters were applied to Pre and Post depolarization conditions. The $\mathrm{CV}^{2}$ ratio was obtained by dividing values before depolarization by those after the step. Note that the important parameter is a $\mathrm{CV}$, but not $\mathrm{CV}^{2}$ as it alone will also enable the comparison.

Statistics are based on paired Student's $t$-test (two-tailed) and the Kolmogorov-Smirnov approach. Comparisons were assumed significant if $P<0.05$.

\section{Solutions and agents}

The ACSF was composed of $119 \mathrm{mM} \mathrm{NaCl}, 2.5 \mathrm{mM} \mathrm{KCl}, 1.25 \mathrm{mM}$ $\mathrm{NaH}_{2} \mathrm{PO}_{4}, 1 \mathrm{mM} \mathrm{MgSO} 4,2.5 \mathrm{mM} \mathrm{CaCl}_{2}, 26 \mathrm{mM} \mathrm{NaHCO}_{3}$, and 10 $\mathrm{mM}$ glucose. By the equilibration of solution with $95 \% \mathrm{O}_{2}$ and $5 \%$
$\mathrm{CO}_{2}$ at room temperature, the $\mathrm{pH}$ of 7.4 was achieved. Only then the solution was used for both the brain slice preparation and recordings. The pipette solution contained $120 \mathrm{mM} \mathrm{K}^{+}$-gluconate, $5 \mathrm{mM} \mathrm{NaCl}, 1 \mathrm{mM} \mathrm{MgCl}$, $10 \mathrm{mM}$ HEPES, $0.2 \mathrm{mM}$ EGTA, $2 \mathrm{mM}$ $\mathrm{Mg}_{2} \mathrm{ATP}, 0.2 \mathrm{mM}$ NaGTP; the $\mathrm{pH}$ of 7.2 was adjusted with $\mathrm{KOH}$. In a set of experiments, the intracellular $\mathrm{Ca}^{2+}$ was chelated with $10 \mathrm{mM}$ EGTA, which was added to a pipette solution. PTX and AM 251 were dissolved in DMSO for the preparation of 100 and $10 \mathrm{mM}$ stock solutions, respectively. Chemicals were purchased from Sigma and Tocris.

\section{Competing interest statement}

S.A.K., K.B., M.W., and B.L have no conflict of interest that could influence this study.

\section{Acknowledgments}

The study was supported by SFB-TRR58 grant from the Deutsche Forschungsgemeinschaft (Fear, Anxiety, and Anxiety Disorders). The skillful technical support was enabled by Martin Purrio and proofreading by Catherine Miller (University of Texas). S.K. is grateful to Dr. Armin Just (University of Freiburg) and Dr. Ludmila Gerashchenko (Chestnut Hill, MA) for reading the latest version.

\section{References}

Aggleton JP, Mishkin M. 1984. Projections of the amygdala to the thalamus in the cynomolgus monkey. J Comp Neurol 222: 56-68. doi:10.1002/cne .902220106

Alger BE, Pitler TA, Wagner JJ, Martin LA, Morishita W, Kirov SA, Lenz RA. 1996. Retrograde signalling in depolarization-induced suppression of inhibition in rat hippocampal CA1 cells. J Physiol 496: 197-209. doi:10 .1113/jphysiol.1996.sp021677

Ali AB. 2007. Presynaptic inhibition of $\mathrm{GABA}_{\mathrm{A}}$ receptor-mediated unitary IPSPs by cannabinoid receptors at synapses between CCK-positive interneurons in rat hippocampus. J Neurophysiol 98: 861-869. doi:10 $.1152 / j n .00156 .2007$

Ameri A. 1999. The effects of cannabinoids on the brain. Prog. Neurobiol 58: 315-348. doi:10.1016/S0301-0082(98)00087-2

Ameri A, Simmet T. 2000. Effects of 2-arachidonylglycerol, an endogenous cannabinoid, on neuronal activity in rat hippocampal slices. Naunyn Schmiedebergs Arch Pharmacol 361: 265-272. doi:10.1007/ s002109900200

Ameri A, Wilhelm A, Simmet T. 1999. Effects of the endogeneous cannabinoid, anandamide, on neuronal activity in rat hippocampal slices. Br J Pharmacol 126: 1831-1839. doi:10.1038/sj.bjp.0702478

Beierlein M, Regehr WG. 2006. Local interneurons regulate synaptic strength by retrograde release of endocannabinoids. J. Neurosci 26: 9935-9943. doi:10.1523/JNEUROSCI.0958-06.2006 
Bliss TVP, Lomo T. 1973. Long-lasting potentiation of synaptic transmission in the dentate area of the anaesthetized rabbit following stimulation of the perforant path. J Physiol 232: 331-356. doi:10.1113/jphysiol.1973 .sp010273

Brown SP, Brenowitz SD, Regehr WG. 2003. Brief presynaptic bursts evoke synapse-specific retrograde inhibition mediated by endogenous cannabinoids. Nat Neurosci 6: 1048-1057. doi:10.1038/nn1126

Bucherelli C, Baldi E, Mariottini C, Passani MB, Blandina P. 2006. Aversive memory reactivation engages in the amygdala only some neurotransmitters involved in consolidation. Learn Mem 13: 426-430. doi:10.1101/lm.326906

Clouston TS. 1870. Observations and experiments on the use of opium, bromide of potassium, and Cannabis indica in insanity, especially in regard to the effects of the two latter given together. Br Foreign Med Chir Rev 46: 493-511.

Devane W, Axelrod J. 1994. Enzymatic synthesis of anandamide, an endogenous ligand for the cannabinoid receptor, by brain membranes. Proc Natl Acad Sci 91: 6698-6701. doi:10.1073/pnas.91.14.6698

Duguid IC, Smart TG. 2004. Retrograde activation of presynaptic NMDA receptors enhances GABA release at cerebellar interneuron-Purkinje cell synapses. Nat Neurosci 7: 525-533. doi:10.1038/nn1227

Faber DS, Korn H. 1991. Applicability of the coefficient of variation method for analyzing synaptic plasticity. Biophys J 60: 1288-1294. doi:10.1016/ S0006-3495(91)82162-2

Fujii S, Yamazaki Y, Goto J-I, Fujiwara H, Mikoshiba K. 2016. Prior activation of inositol 1,4,5-trisphosphate receptors suppresses the subsequent induction of long-term potentiation in hippocampal CA1 neurons. Learn Mem 23: 208-220. doi:10.1101/lm.041053.115

Fujii S, Yamazaki Y, Goto JI, Fujiwara H, Mikoshiba K. 2020. Depotentiation depends on $\mathrm{IP}_{3}$ receptor activation sustained by synaptic inputs after LTP induction. Learn Mem 27: 52-66. doi:10.1101/lm.050344.119

Hamill OP, Marty A, Neher E, Sakmann B, Sigworth FJ. 1981. Improved patch-clamp techniques for high-resolution current recording from cells and cell-free membrane patches. Pflugers Arch 391: 85-100. doi:10 .1007/BF00656997

Herkenham M, Lynn A, Little M, Johnson M, Melvin L, de Costa B, Rice K. 1990. Cannabinoid receptor localization in brain. Proc Natl Acad Sci 87: 1932-1936. doi:10.1073/pnas.87.5.1932

Hill EL, Gallopin T, Ferezou I, Cauli B, Rossier J, Schweitzer P, Lambolez B. 2007. Functional CB1 receptors are broadly expressed in neocortical GABAergic and glutamatergic neurons. J Neurophysiol 97: 2580-2589. doi:10.1152/jn.00603.2006

Hill MN, McLaughlin RJ, Morrish AC, Viau V, Floresco SB, Hillard CJ, Gorzalka BB. 2009. Suppression of amygdalar endocannabinoid signaling by stress contributes to activation of the hypothalamic-pituitary-adrenal axis. Neuropsychopharmacology 34: 2733-2745. doi:10.1038/npp.2009.114

Ivanova SY, Storozhuk MV. 2011. Post-tetanic and depolarization-induced suppression of inhibition in hippocampal cell cultures: are similar mechanisms involved? Neurophysiology 43: 209-212. doi:10.1007/ s11062-011-9207-3

Kano M, Ohno-Shosaku T, Hashimotodani Y, Uchigashima M, Watanabe M. 2009. Endocannabinoid-mediated control of synaptic transmission. Physiol Rev 89: 309-380. doi:10.1152/physrev.00019.2008

Kodirov SA, Takizawa S, Joseph J, Kandel ER, Shumyatsky GP, Bolshakov VY. 2006. Synaptically released zinc gates long-term potentiation in fear conditioning pathways. Proc Natl Acad Sci 103: 15218-15223. doi:10 $.1073 /$ pnas.0607131103
Kodirov SA, Jasiewicz J, Amirmahani P, Psyrakis D, Bonni K, Wehrmeister M, Lutz B. 2010. Endogenous cannabinoids trigger the depolarizationinduced suppression of excitation in the lateral amygdala. Learn Mem 17: 43-49. doi:10.1101/lm.1663410

Krishtal O. 2015. Receptor for protons: first observations on acid sensing ion channels. Neuropharmacology 94: 4-8. doi:10.1016/j.neuropharm.2014 .12 .014

Lemak MS, Bravarenko NI, Bobrov MY, Bezuglov VV, Ierusalimsky VN, Storozhuk MV, Malyshev AY, Balaban PM. 2007. Cannabinoid regulation in identified synapse of terrestrial snail. Eur J Neurosci 26: 3207-3214. doi:10.1111/j.1460-9568.2007.05945.x

Lemtiri-Chlieh F, Levine E. 2007. Lack of depolarization-induced suppression of inhibition (DSI) in layer $2 / 3$ interneurons that receive cannabinoid-sensitive inhibitory inputs. J Neurophysiol 98: 2517-2524. doi:10.1152/jn.00817.2007

Llano I, Leresche N, Marty A. 1991. Calcium entry increases the sensitivity of cerebellar Purkinje cells to applied GABA and decreases inhibitory synaptic currents. Neuron 6: 565-574. doi:10.1016/0896-6273(91) 90059-9

Medina I, Leinekugel X, Ben-Ari Y. 1999. Calcium-dependent inactivation of the monosynaptic NMDA EPSCs in rat hippocampal neurons in culture. Eur J Neurosci 11: 2422-2430. doi:10.1046/j.1460-9568.1999 $.00664 . \mathrm{x}$

Melamede R. 2009. Parasitic brain infection, endocannabinoids, and schizophrenia. Med Hypotheses 72: 220-222. doi:10.1016/j.mehy.2008 .08 .025

Patel S, Kingsley PJ, Mackie K, Marnett LJ, Winder DG. 2009. Repeated homotypic stress elevates 2-arachidonoylglycerol levels and enhances short-term endocannabinoid signaling at inhibitory synapses in basolateral amygdala. Neuropsychopharmacology 34: 2699-2709. doi:10 .1038/npp.2009.101

Pavlov IP. 1927. Conditioned reflexes. Dover, New York.

Pertwee RG. 2006. Cannabinoid pharmacology: the first 66 years. $\mathrm{Br} \mathrm{J}$ Pharmacol 147: S163-S171. doi:10.1038/sj.bjp.0706406

Racine RJ, Milgram NW, Hafner S. 1983. Long-term potentiation phenomena in the rat limbic forebrain. Brain Res 260: 217-231. doi:10 .1016/0006-8993(83)90676-5

Rogan MT, Staubli UV, LeDoux JE. 1997. Fear conditioning induces associative long-term potentiation in the amygdala. Nature 390: 604607. doi:10.1038/37601

Urbanski MJ, Kovacs FE, Szabo B. 2009. Depolarizing GABAergic synaptic input triggers endocannabinoid-mediated retrograde synaptic signaling. Synapse 63: 643-652. doi:10.1002/syn.20641

Wilson RI, Kunos G, Nicoll RA. 2001. Presynaptic specificity of endocannabinoid signaling in the hippocampus. Neuron 31: 453-462. doi:10.1016/S0896-6273(01)00372-5

Yoshida T, Uchigashima M, Yamasaki M, Katona I, Yamazaki M, Sakimura K, Kano M, Yoshioka M, Watanabe M. 2011. Unique inhibitory synapse with particularly rich endocannabinoid signaling machinery on pyramidal neurons in basal amygdaloid nucleus. Proc Natl Acad Sci 108 3059-3064. doi:10.1073/pnas.1012875108

Zhu PJ, Lovinger DM. 2005. Retrograde endocannabinoid signaling in a postsynaptic neuron/synaptic bouton preparation from basolateral amygdala. J Neurosci 25: 6199-6207. doi:10.1523/JNEUROSCI.1148-05 .2005

Received September 13, 2020; accepted in revised form October 13, 2020. 


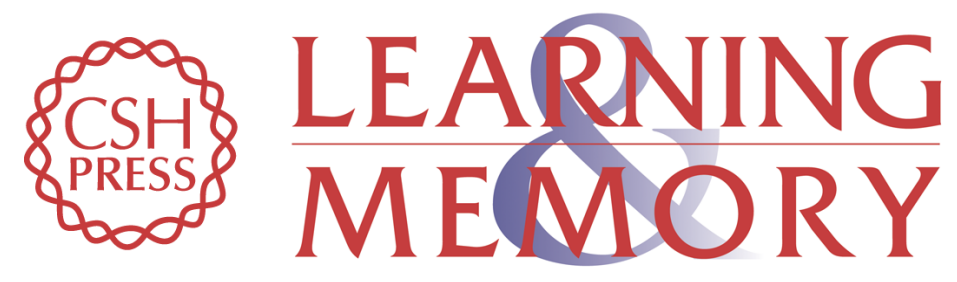

\section{Depolarization-initiated endogenous cannabinoid release and underlying retrograde neurotransmission in interneurons of amygdala}

Sodikdjon A. Kodirov, Kathrin Bonni, Michael Wehrmeister, et al.

Learn. Mem. 2021, 28:

Access the most recent version at doi:10.1101/lm.052555.120

References This article cites 38 articles, 10 of which can be accessed free at: http://learnmem.cshlp.org/content/28/2/44.full.html\#ref-list-1

Creative This article is distributed exclusively by Cold Spring Harbor Laboratory Press for the Commons first 12 months after the full-issue publication date (see

License http://learnmem.cshlp.org/site/misc/terms.xhtml). After 12 months, it is available under a Creative Commons License (Attribution-NonCommercial 4.0 International), as described at http://creativecommons.org/licenses/by-nc/4.0/.

Email Alerting Receive free email alerts when new articles cite this article - sign up in the box at the Service top right corner of the article or click here. 